\title{
The incidence of hypomagnesaemia following abdominal aortic aneurysm surgery
}

\author{
K Jolly, R Faulconer, R McEwan, H Becker, A Garnham
}

Dudley Group NHS Foundation Trust, UK

\section{ABSTRACT}

INTRODUCTION Magnesium is important for cardiac function. Hypomagnesaemia is associated with a higher incidence of arrhythmias and poorer outcomes in cardiac surgery. No studies have investigated the incidence or impact of postoperative hypomagnesaemia after abdominal aortic aneurysm (AAA) surgery. We aim to assess the incidence of hypomagnesaemia after AAA repair in our population.

METHODS Retrospective analysis was performed of patients who underwent elective AAA surgery at a single vascular centre. The last 110 patients undergoing open or endovascular AAA repair were identified. The hospital pathology system was used to identify the immediate postoperative serum magnesium levels as well as patient demographics and admission details. Hypomagnesaemia was defined as serum magnesium of $<0.7 \mathrm{mmol} / \mathrm{l}$.

RESULTS A total of 211 patients were studied and there were 3 deaths. Of the patients included, 101 underwent open elective AAA repair and 110 underwent endovascular repair. In the elective open repair group, 73 patients (73\%) were hypomagnesaemic. In the endovascular repair group, $35(32 \%)$ had hypomagnesaemia. A t-test showed a statistically significant difference in hypomagnesaemia between the open and endovascular groups $(p<0.001)$.

CONCLUSIONS AAA surgery is associated with a high incidence of postoperative hypomagnesaemia, which is significantly greater among open repair patients. This is likely to have an effect on cardiac activity and lead to cardiac complications such as arrhythmias and poorer postoperative outcomes, especially in the open AAA repair subgroup. This stresses the importance of serum magnesium and cardiac monitoring in the postoperative phase. A prospective study is proposed to further investigate these findings, and their potential implications on perioperative morbidity and mortality.

KEYWORDS

Abdominal aortic aneurysm - Hypomagnesaemia - Arrhythmias - Endovascular surgery - Vascular anaesthesia

Accepted 20 January 2015

CORRESPONDENCE TO

Karan Jolly, E: kjolly@nhs.net

Magnesium is an important cofactor of the sodium-potassium ATPase pump helping to maintain the myocyte resting potential, making it a very important electrolyte with regard to cardiac function. Experiments have demonstrated a change in cardiac tissue electrophysiology by extracellular magnesium alteration, and hypomagnesaemia has been associated with an increased risk of aberrant myocardial activity and subsequent cardiac arrhythmias. ${ }^{1-7}$ Furthermore, patients with hypomagnesaemia have a higher incidence of life threatening arrhythmias following myocardial infarction. ${ }^{8}$ Use of intravenous infusion of magnesium has been shown to decrease fatal consequences and mortality following arrhythmias in these patients. This suggests that serum magnesium levels may be of even more importance in patients with established cardiovascular disease.

Serum magnesium levels are not routinely monitored preoperatively in most elective procedures. The prevalence of hypomagnesaemia in the general population is estimated to be around $7 \%$ but is significantly higher $(60 \%)$ in a postoperative intensive care setting. ${ }^{9}$ Measuring preoperative magnesium levels has been suggested in high risk patients including those on predisposing drugs (diuretics, aminoglyclosides, ciclosporin), hypertensive patients, alcoholics, patients with structural heart disease and diabetic patients who have increased renal losses. ${ }^{10-12}$ These patients are at higher risk of hypomagnesaemia following major surgery, and subsequent morbidity and mortality from ventricular tachyarrhythmias. ${ }^{13}$

Several studies have identified that hypomagnesaemia is common following cardiac surgery. They have demonstrated a significant fall in serum magnesium levels in patients undergoing cardiac procedures such as coronary artery bypass grafting (CABG), resulting in adverse cardiac and non-cardiac outcomes among hypomagnesaemic patients. ${ }^{14-16}$ The mechanism behind this process is not fully understood. One mechanism proposed is that of haemodilution with prime fluid in the extracorporeal circuit in addition to intravenous fluid replacement. However, this is 
not the sole explanation as concentrations of other electrolytes and proteins do not fall by the same magnitude. ${ }^{17-19}$

Another mechanism proposed is of beta-adrenergic mediated lipolysis in response to stress and increased fatty acid circulation. This leads to a fall in intracellular ionised magnesium levels following precipitation of insoluble magnesium soaps. ${ }^{20-22}$ Patients with abdominal aortic aneurysms (AAAs) have similar cardiovascular disease risk factors to those with coronary artery disease. This immediately puts them at higher risk of hypomagnesaemia, as discussed previously, especially following major vascular surgery. ${ }^{13}$ Despite this, no studies have been conducted to assess the incidence of hypomagnesaemia following AAA surgery (open repair [OR] or endovascular repair [EVAR]).

While working in a regional vascular unit, we came across a large proportion of OR patients who had hypomagnesaemia postoperatively. Likewise, a number of patients who developed a tachyarrhythmia following surgery were found to be hypomagnesaemic and were managed successfully with magnesium replacement. As a result, based on our clinical findings and in light of previously published data, we hypothesised that hypomagnesaemia is a common occurrence following AAA repair. We therefore conducted a retrospective analysis to quantify the incidence of hypomagnesaemia in patients undergoing AAA surgery. Furthermore, we tried to identify any difference between patients undergoing OR or EVAR.

\section{Methods}

Data were collected from a single UK regional vascular surgery centre. The last 110 patients undergoing OR or EVAR were identified using the trust database. Patient demographics, blood test results and admission details were obtained from the electronic records, and a retrospective analysis was performed. The first postoperative magnesium level was noted, which is obtained within an hour of arriving on a high dependency or intensive care unit. A level of $0.7 \mathrm{mmol} / \mathrm{l}$ was considered the lower limit of normal as per local guidelines. The focus of this study was the incidence of immediate postoperative hypomagnesaemia. Preoperative magnesium levels are not measured routinely for most procedures and so it was not possible to obtain these results to make a pre/postoperative comparison.

SPSS $^{\circledR}$ version 17.0 (SPSS, Chicago, IL, US) was used to conduct all statistical analyses including for descriptive and parametric statistics. Any differences between groups were deemed statistically significant at a $p$-value of $<0.05$.

\section{Results}

Of the 220 patients identified, 9 were excluded in the OR group owing to coding errors. Data were therefore analysed for 211 patients: 101 OR and 110 EVAR. In the OR group, ages ranged from 51 to 86 years (mean: 71 years) with 21 female and 80 male subjects. In the EVAR group, ages ranged from 62 to 92 years (mean: 76 years) with 9 female and 101 male subjects. Both groups were similar with regard to the proportion of male to female subjects, with the EVAR group having a slightly higher mean age.
In the OR group, 73/101 patients (73\%) had hypomagnesaemia postoperatively on arrival on the ward while $35 / 110$ subjects (32\%) had hypomagnesaemia in the EVAR group. An independent samples t-test was conducted to compare serum magnesium levels between the two groups. Results of the analysis revealed that magnesium levels for patients undergoing OR (mean: $0.67 \mathrm{mmol} / \mathrm{l}$, standard deviation [SD]: $0.16 \mathrm{mmol} / \mathrm{l}$ ) were lower than for patients undergoing EVAR (mean: $0.74 \mathrm{mmol} / \mathrm{l}$, SD: $0.11 \mathrm{mmol} / \mathrm{l})$. This difference was statistically significant $(\mathrm{t}(173.87)=-3.372, p<0.001)$.

The median length of stay in the OR group was 9 days among both the hypomagnesaemic and normomagnesaemic patients. The median length of stay in the EVAR group was less at 4 days. There was no discernable correlation between serum magnesium levels and length of stay, as with the OR group. A total of three inpatient deaths were reported. All three patients were in the OR group and were hypomagnesaemic following surgery. The cause of death was hospital acquired pneumonia in two cases and myocardial infarction in one case. Table 1 demonstrates the distribution of serum magnesium levels in each group.

\section{Discussion}

Elective AAA surgery appears to be associated with a high incidence of postoperative hypomagnesaemia, as with cardiac surgery. Our results show that between the two well established modalities of elective AAA repair, there is a significantly higher proportion of hypomagnesaemic patients in the OR group ( $73 \%$ vs $32 \%$ ).

Several studies have shown a causative relationship between postoperative hypomagnesaemia and arrhythmias after CABG. One such study by Aglio et al looked at 101 patents undergoing cardiac surgery $\left(70 \%\right.$ CABG). ${ }^{15}$ Of these, $19.2 \%$ of patients had low serum magnesium prior to surgery compared with $71 \%$ immediately after surgery. There were significantly more episodes of atrial arrhythmias and prolonged ventilatory support $(34.9 \%$ vs $21.4 \%$, $p<0.05)$ in the group of hypomagnesaemic patients.

Similar observational studies have shown mean serum magnesium levels to be significantly lower in patients who develop postoperative atrial fibrillation (AF) after CABG. ${ }^{14,23}$ However, there are conflicting views with respect to a causal

\begin{tabular}{|c|c|c|}
\hline \multirow{2}{*}{$\begin{array}{l}\text { Table } 1 \\
\text { group }\end{array}$} & \multicolumn{2}{|c|}{ Type of repair } \\
\hline & Open & Endovascular \\
\hline Patients & 101 & 110 \\
\hline Mean (SD) & $\begin{array}{l}0.67 \mathrm{mmol} / \mathrm{l} \\
\text { (SD: } 0.16 \mathrm{mmol} / \mathrm{l})\end{array}$ & $\begin{array}{l}0.74 \mathrm{mmol} / \mathrm{l} \\
\text { (SD: } 0.11 \mathrm{mmol} / \mathrm{l})\end{array}$ \\
\hline Median (IQR) & $\begin{array}{l}0.64 \mathrm{mmol} / \mathrm{l} \\
(0.59-0.70 \mathrm{mmol} / \mathrm{l})\end{array}$ & $\begin{array}{l}0.74 \mathrm{mmol} / \mathrm{l} \\
(0.68-0.78 \mathrm{mmol} / \mathrm{l})\end{array}$ \\
\hline
\end{tabular}


relationship. A review by Archbold and Zaman looked at the use of magnesium for the prevention of AF following CABG. ${ }^{24}$ Although analysis of several studies seems to highlight an association between hypomagnesaemia and postoperative AF, the review failed to show significant efficacy of magnesium therapy in AF prevention after CABG. The inconclusive findings of these trials were partly due to limitations in methodology but most probably reflect the poor correlation between serum magnesium and intracellular (especially intra-atrial) magnesium levels.

The postoperative incidence of $\mathrm{AF}$ in patients undergoing AAA repair has been documented as $10-20 \% .^{23,25-27}$ In these studies, serum magnesium levels have not been analysed as a potential precipitant to the arrhythmia. Until now, no association has been made between new onset arrhythmias and hypomagnesaemia after AAA surgery. A study conducted by Feringa et al in 2007 aimed to examine differences in postoperative arrhythmias, myocardial ischaemia and cardiac events between patients undergoing elective OR and EVAR. ${ }^{28}$ Their results showed that EVAR was associated with a lower incidence of arrhythmias, myocardial ischaemia, troponin $\mathrm{T}$ release and cardiac events than OR. Prevalence of arrhythmias in the EVAR group was $14 \%$ compared with $29 \%$ in the OR group $(p=0.04)$. Whether this is related to the findings of a higher incidence of hypomagnesaemia in the OR group is yet to be proven.

\section{Conclusions}

Our results suggest a high incidence of postoperative hypomagnesaemia among patients undergoing elective AAA surgery. The degree of hypomagnesaemia was significantly greater in the OR group. The exact cause of this fall in serum magnesium is not entirely clear. Similar to cardiac surgery, it may be associated with higher rates of cardiac and non-cardiac complications (arrhythmias, myocardial ischaemia, prolonged ventilation, infection etc) in the hypomagnesaemic group.

Cardiac complications continue to be a major cause of morbidity and mortality among patients treated with vascular surgery, and the effect of hypomagnesaemia on this is yet to be proven. Unfortunately, owing to limitations with record keeping, it was not possible to comment on postoperative cardiac complications and perioperative magnesium administration for these patients. Consequently, as a follow-up to these findings, we intend to design a multicentre prospective study in an attempt to identify any association between postoperative AAA hypomagnesaemia and both cardiac/non-cardiac complications. Lastly, it should be stressed that serum magnesium and cardiac activity should be monitored closely in the early postoperative phase in all patients undergoing AAA repair, and normal serum magnesium levels maintained.

\section{References}

1. Iseri LT, Allen BJ, Ginkel ML, Brodsky MA. Ionic biology and ionic medicine in cardiac arrhythmias with particular reference to magnesium. Am Heart J 1992; 123: $1,404-1,409$.
2. Kulick DL, Hong R, Ryzen E et al. Electrophysiologic effects of intravenous magnesium in patients with normal conduction systems and no clinical evidence of significant cardiac disease. Am Heart J 1988; 115: 367-373.

3. Hollifield JW. Magnesium depletion, diuretics, and arrhythmias. Am J Med 1987; 82: 30-37.

4. DiCarlo LA, Morady F, de Buitleir M et al. Effects of magnesium sulfate on cardiac conduction and refractoriness in humans. J Am Coll Cardiol 1986; 7 1,356-1,362.

5. Dyckner T. Serum magnesium in acute myocardial infarction. Relation to arrhythmias. Acta Med Scand 1980; 207: 59-66.

6. Woods WT, Katholi RE, Urthaler F, James TN. Electrophysiological effects of magnesium on cells in the canine sinus node and false tendon. Circ Res 1979; 44: 182-188.

7. Watanabe Y, Dreifus LS. Electrophysiological effects of magnesium and its interactions with potassium. Cardiovasc Res 1972; 6: 79-88.

8. Rasmussen HS, McNair P, Norregard $\mathrm{P}$ et al. Intravenous magnesium in acute myocardial infarction. Lancet 1986; 1: 234-236.

9. Whang R, Hampton EM, Whang DD. Magnesium homeostasis and clinical disorders of magnesium deficiency. Ann Pharmacother 1994; 28: 220-226.

10. Fawcett WJ, Haxby EJ, Male DA. Magnesium: physiology and pharmacology. Br J Anaesth 1999; 83: 302-320.

11. Agus ZS. Hypomagnesemia. J Am Soc Nephrol 1999; 10: 1,616-1,622.

12. White JR, Campbell RK. Magnesium and diabetes: a review. Ann Pharmacother 1993; 27: 775-780.

13. Sheybani A, Geraci SA. When should serum magnesium be measured prior to non-cardiac surgery? J Miss State Med Assoc 2008; 49: 295-298.

14. Zaman AG, Alamgir $F$, Richens $T$ et al. The role of signal averaged $P$ wave duration and serum magnesium as a combined predictor of atrial fibrillation after elective coronary artery bypass surgery. Heart 1997; 77: 527-531.

15. Aglio LS, Stanford GG, Maddi R et al. Hypomagnesemia is common following cardiac surgery. J Cardiothorac Vasc Anesth 1991; 5: 201-208.

16. Scheinman MM, Sullivan RW, Hyatt KH. Magnesium metabolism in patients undergoing cardiopulmonary bypass. Circulation 1969; 39(5 Suppl 1): I235-I241.

17. Bunton RW. Value of serum magnesium estimation in diagnosing myocardial infarction and predicting dysrhythmias after coronary artery bypass grafting. Thorax 1983; 38: 946-950.

18. Westhorpe RN, Varghese Z, Petrie A et al. Changes in ionized calcium and other plasma constituents associated with cardiopulmonary bypass. Br J Anaesth 1978; 50: 951-957.

19. Holden MP, Ionescu MI, Wooler GH. Magnesium in patients undergoing open-heart surgery. Thorax 1972; 27: 212-218.

20. Reinhart RA. Magnesium metabolism. A review with special reference to the relationship between intracellular content and serum levels. Arch Intern Med 1988; 148: 2,415-2,420.

21. Whyte KF, Addis GJ, Whitesmith R, Reid JL. Adrenergic control of plasma magnesium in man. Clin Sci 1987; 72: 135-138.

22. Flink EB, Brick JE, Shane SR. Alterations of long-chain free fatty acid and magnesium concentrations in acute myocardial infarction. Arch Intern Med 1981: 141: 441-443.

23. Kalman JM, Munawar M, Howes LG et al. Atrial fibrillation after coronary artery bypass grafting is associated with sympathetic activation. Ann Thorac Surg 1995; 60: 1,709-1,715.

24. Archbold RA, Zaman AG. Magnesium for atrial fibrillation after coronary artery bypass graft surgery: its role in aetiology and prevention. Crit Care Resusc 2000; 2: 260-268

25. Noorani A, Walsh SR, Tang TY et al. Atrial fibrillation following elective open abdominal aortic aneurysm repair. Int J Surg 2009; 7: 24-27.

26. Perzanowski C, Gandhi S, Pai RG. Incidence and predictors of atrial fibrillation after aortic repairs. Am J Cardiol 2004; 93: 928-930.

27. Valentine RJ, Rosen SF, Cigarroa JE et al. The clinical course of new-onset atrial fibrillation after elective aortic operations. J Am Coll Surg 2001; 193 499-504.

28. Feringa $\mathrm{HH}$, Karagiannis $\mathrm{S}$, Vidakovic $\mathrm{R}$ et al. Comparison of the incidences of cardiac arrhythmias, myocardial ischemia, and cardiac events in patients treated with endovascular versus open surgical repair of abdominal aortic aneurysms. Am J Cardiol 2007; 100: 1,479-1,484. 